\title{
L'inversion pronominale : histoire et analyse
}

\author{
Jesse Tseng \\ CLLE-ERSS UMR 5263 \\ CNRS \& Université de Toulouse \\ Jesse.Tseng@univ-tlse2.fr
}

Cet exposé est consacré à la description et à l'analyse de l'inversion pronominale (« inversion clitique ») que l'on trouve surtout dans les propositions principales interrogatives, mais également dans les incises, dans les propositions introduites par certaines locutions adverbiales, et dans une moindre mesure dans les propositions conditionnelles et quelques autres formules littéraires.

- Qui êtes-vous? Devrais-je tout dire?

- Je vous déteste, dit-il / s'écria-t-elle.

- Peut-être pourras-tu venir plus tôt. Sans doute leur avons-nous trop demandé.

- Seraient-ils restés que nous les aurions vus. Ne fût-ce qu'un instant.

L'inversion du sujet nominal («inversion stylistique ») est possible dans certains de ces contextes (interrogatives $q u$-, incises), mais cette construction ne présente pas du tout les mêmes propriétés et ne sera pas examinée en détail ici.

Nous ne donnons ici que l'inventaire des types de phrases admettant l'inversion pronominale, sans essayer d'expliquer cette distribution. Nous examinerons plutôt la structure interne de la construction, et quelques aspects de son histoire :

- l'évolution diachronique du $t$ épenthétique

- les propriétés phono-syntaxiques des structures inversées

- l'analyse syntaxique de l'inversion complexe

\section{L'histoire du $t$ épenthétique}

Dans les structures inversées, les pronoms de la $3^{\mathrm{e}}$ pers. à initiale vocalique $(\mathrm{il}(\mathrm{s})$, elle $(s)$, on) sont obligatoirement liées au verbe par la consonne [t]. Si le verbe se termine orthographiquement par une consonne dentale, le pronom inversé est lié simplement par un trait d'union. C'est le cas de tous les pluriels (en «nt ») et des singuliers en « $\mathrm{t} »:$ : dorment-ils, dort-elle, dormait-on ». Les singuliers en « $\mathrm{d}$ » suivent la même règle : «vend-elle, mord-il». En effet, ce « $\mathrm{d}$ » n'a jamais eu d'autre valeur phonétique que $[\mathrm{t}]$ : Les savants du Moyen Âge ont remplacé le « $\mathrm{t}$ » final étymologique de ces formes par le $\langle\mathrm{d} » \mathrm{du}$ radical présent ailleurs dans le paradigme. Pour les formes verbales a finale vocalique (y compris $e$ muet), la soudure consonantique doit être notée explicitement «-t-». Cela concerne principalement les formes du présent en «e » et celles du passé simple et du futur en «-a »: « aime-t-il, alla-t-on, ira-t-on ». Dans cette section, nous étudierons les origines de cette pratique et les conséquences des considérations diachroniques pour l'analyse du français moderne.

\subsection{L'évolution des formes verbales}

Pour la plupart des formes verbales en « $\mathrm{t} / \mathrm{d} »$, la consonne finale graphique représente un [t] qui remonte à l'époque latine et qui n'a jamais cessé d'être prononcé dans ces combinaisons (et dans d'autres contextes de liaison, c.-à-d. devant un mot à initiale vocalique). Les principales exceptions sont certains passés simples en « it» et en «ut»; dans ces formes le $t$ final a disparu régulièrement avant la fin du $\mathrm{XI}^{\mathrm{e}}$ siècle (graphiquement : «perdi, fu », et sans doute phonétiquement), mais il a été restitué entre le XIII et 
le $\mathrm{XVI}^{\mathrm{e}}$ siècles sur le modèle de formes comme «fit, vit», qui ont toujours gardé leur $t$. Par manque de modèle analogique, cette restitution $\mathrm{du} \ll \mathrm{t} » \mathrm{n}$ 'a pas touché aux passés simples en «a $»$.

Bien entendu, les formes de la $3^{\mathrm{e}}$ pers. sing. en «e » et en «a » correspondent également à des formes latines en $t$ (portat, portā $(v i) t$, etc.), et on affirme parfois que c'est ce $t$ primitif qui est conservé dans les inversions («porte-t-il »). Les faits historiques montrent clairement que ce n'est pas le cas. Il faut considérer deux groupes de formes verbales. Dans le premier groupe, le $t$ final suit la voyelle directement; il n'est pas appuyé par une autre consonne (ou semi-consonne). Le $t$ de ces formes s'est d'abord affaibli (en $\theta$ ) et ensuite amuï, avant la fin du XI $\mathrm{X}^{\mathrm{e}}$ siècle :

- $\quad$ ortat $>$ porte $\theta>$ porte, $\operatorname{porta}(v i) t>\operatorname{porta} \theta>$ porta

Les mêmes remarques s'appliquent aux formes du futur en - $a$ (désinence dérivant de l'auxiliaire $a t$ ). Dans un premier temps, l'orthographe, plus conservatrice, ne reflète pas systématiquement cet effacement consonantique. Dans les textes du XII ${ }^{\mathrm{e}}$ siècle, il y a beaucoup d'hésitation entre les graphies avec et sans «-t », mais à partir du siècle suivant, la consonne n'est plus du tout notée à la fin des formes verbales en question. La scansion des vers de cette période ( $\mathrm{du} \mathrm{XIII}^{\mathrm{e}}$ au XV $\mathrm{XV}^{\mathrm{e}}$ siècle) confirme que même en contexte de liaison, le $t$ primitif de ces formes a été totalement éliminé. Par exemple, les séquences comme aime il et nomme on peuvent compter pour deux syllabes, ce qui indique non seulement l'absence d'une consonne finale mais également l'élision de la voyelle centrale : aim'il, nomm'on. Dans les exemples sans élision (alla on), rien ne peut indiquer la présence d'une consonne (étymologique ou épenthétique) au lieu d'un hiatus parfaitement toléré à cette époque.

Dans l'autre groupe de formes verbales, le $t$ final était en contact avec une autre consonne ou une diphtongue :

- dormit $>$ dor $(m) t$, facit $>$ fait, portant $>$ portent

Ainsi protégée, la consonne a survécu à la première vague d'effacements (du IXe au XIe) et n'a commencé à s'amuïr qu'à partir de la seconde moitié du XII ${ }^{\mathrm{e}}$ siècle, prise dans une évolution très longue qui a fini par effacer la majorité des consonnes finales du français. Elles ont disparu progressivement devant un autre mot à initiale consonantique, et ensuite à la pause. La langue savante s'est opposée à ce changement, et a même réussi à le renverser dans certains cas, mais pas dans les formes verbales en question. Ici, le $t$ final — toujours noté graphiquement («dort, fait, portent ») — a disparu définitivement au XVII ${ }^{\mathrm{e}}$ siècle, sauf en contexte de liaison.

\subsection{La réintroduction du $t$}

Holbrook (1923) relève, dans une chronique datant du milieu du $\mathrm{XV}^{\mathrm{e}}$ siècle, une des premières attestations du $t$ intercalé : «Cuide ton [croit-on] que je sois Vauldoise ? ». Cette prononciation, d'abord typique de la langue vulgaire, s'est généralisée par la suite. Dans la Gramère de Ramus (1562), on lit : «Pour ne point tomber en déplaisante concurrence de voyelles, nous interposons quelquefois ung $t[\ldots]$ comme at $i$ faict » (cité par Delattre 1955). À cette époque, les graphies avec « $\mathrm{t} »$ rencontraient encore beaucoup de résistance: Peletier du Mans, dans son Dialogue de l'ortografe e prononciation françoese (1550), explique que les séquences « dine il » et « ira il » se prononcent dine ti et ira ti, mais qu'il «seroit chose ridicule si nous les escrivions selon qu'ils se prononcent ». Mais un siècle plus tard, Vaugelas n'admet que la graphie ridicule prie-t-on, alla-t-on «pour oster la cacophonie», et il condamne la prononciation sans $t$ des séquences alla-il, alla-on (la norme savante encore deux siècles auparavant).

Nous pouvons expliquer le développement de ce $t$ comme un cas de réanalyse. Le fait que $t$ continuait à être prononcé systématiquement dans les combinaisons fait il, est on, alors qu'il s'amuïssait progressivement dans les mêmes mots dans beaucoup d'autres contextes, a renforcé le lien entre le verbe et le sujet pronominal enclitique. Ce qui était au départ un cas de liaison régulière a fini par acquérir un statut grammatical particulier. En français moderne, on observe que ces verbes font plutôt rarement la liaison dans d'autres combinaisons, et que pour certains verbes la réalisation du [t] n'est plus du tout possible : «*Elle dort [t]à la maison». En revanche, le [t] dans les structures inversées est obligatoire. La 
grammaticalisation des combinaisons verbe + pronom enclitique a conduit à une réanalyse $d u[t]$ dans cette construction. Il a cessé d'être une consonne de liaison, pour devenir la consonne initiale d'une forme spéciale du pronom :

- [ti(l)], [tEl], [tõ(n)]

Cet allomorphe du pronom enclitique s'est généralisé aux structures avec les autres verbes, en «e/a »: aime-[ti(l)] et alla-[tõ(n)].

La réanalyse pronominale fournit une explication générale de l'introduction du $t$ «épenthétique ». La généralisation des formes en $t$ - a simplifié le paradigme des pronoms inversés au singulier, en éliminant les formes [i(l)], [El] et [õ(n)]. Elle a également rendu plus uniforme l'inventaire des pronoms de la $3^{\mathrm{e}}$ pers., qui commençaient désormais tous par $t^{-}{ }^{l}$. Rappelons qu'au pluriel, les verbes se terminaient tous par $t$, permettant l'hypothèse d'un allomorphe pronominal [ti(l)(z)] ou [tEl(z)] dans tous les cas. La tendance à la réduction des hiatus en moyen français a pu jouer un rôle dans un cas comme alla-on $>$ alla-t-on, d'où l'appellation traditionnelle «t euphonique (ou)». Pour un cas comme aime il, cette explication est moins convaincante, vu que l'hiatus était déjà évité par l'élision de $e$. Mais dans la syllabation de aim'il [emil], la consonne du radical verbal est associée au pronom, ce qui peut entraver (légèrement) l'intelligibilité de l'énoncé. Dans la réalisation aime-t-il [Em(ə)til], intégrité phonologique du radical verbal est assurée. Ces considérations phonologiques sont relativement mineures, et la motivation principale reste morphologique : l'uniformisation du paradigme des pronoms inversés.

Le développement historique des formes verbales en «e/a » montre que le $t$ dans aime-t-il n'est pas le continuateur direct d'un $t$ primitif latin. On peut aussi écarter l'hypothèse d'une restitution de consonne finale. Les formes verbales auxquelles on a restitué une consonne de liaison à partir du XIII ${ }^{\mathrm{e}}$ siècle ont vu modifier leur graphie :

- «dormi, perdi, fu » $\rightarrow$ « dormit, perdit, fut»

Les consonnes finales s'amuïssaient à la même époque, mais ce $t$ restitué est réapparu dans tous les contextes de liaison. Ce n'est pas le cas des verbes en «e/a», qui ont perdu définitivement leur consonne de liaison au XI ${ }^{\mathrm{e}}$ siècle $^{2}$. Dans aime-t-il, alla-t-elle, donc, le $[\mathrm{t}]$ ne fait pas partie de la structure phonologique du verbe.

\subsection{D'autres signes de grammaticalisation}

L'insertion obligatoire de [t] (et de «-t-» dans l'orthographe) à la $3^{\mathrm{e}}$ personne est aujourd'hui le signe le plus visible du lien étroit qui existe entre le verbe et le pronom dans les structures inversées. D'autres effets ont pu se manifester, sans être adoptés définitivement. $A u X V^{\mathrm{e}}$ siècle, les formes de la $2^{\mathrm{e}}$ pers. plur. «avez-vous, savez-vous» ont été contractées en «av'ous, sav'ous» (graphiés parfois «a^uous» avec l'accent circonflexe, dont l'usage était loin d'être fixé à cette époque). À la $1^{\text {re }}$ pers. sing., l'enclise de -je aux verbes en $e$ final est devenue problématique au $\mathrm{XV}^{\mathrm{e}}$ siècle : l'univerbation d'une séquence comme aime-je $(<$ áime je) a entraîné une modification phonologique, l'accent ne pouvant pas rester sur l'antépénultième. La solution adoptée - l'ouverture de l'e désinentiel en é ou en è accentué («aimé-je, aimè-je ») - est aujourd'hui tombée en désuétude. Les verbes se terminant par une voyelle nasale ou par une consonne ont également posé problème aux locuteurs, et aucune forme alternative (par ex. «prétendé-je » pour «prétends-je ») n'a reçu l'approbation des grammairiens. Depuis le XVII ${ }^{\mathrm{e}}$ siècle, c'est la tournure en « est-ce que » qui est préférée. La disparition des séquences inversées montre qu'il ne s'agissait plus d'une combinaison syntaxique libre.

On peut mentionner enfin que la soudure du verbe et du pronom inversé contraste avec ce que l'on observe dans l'inversion stylistique :

- Combien de livres lit [t] un bon élève?

- Je suis là, dit $[t]$ une voix. 
Si la liaison est possible dans un style soutenu, elle n'a pas du tout le même statut que le [t] obligatoire de l'inversion pronominale. Selon le cadre analytique adopté, le sujet nominal doit occuper une position distincte (même en l'absence d'un complexe verbal, comme dans les exemples ci-dessus), ou bien il faut deux mécanismes distincts pour la combinaison d'un verbe avec un sujet inversé. Dans certaines analyses, par exemple, le sujet nominal inversé est traité comme un COD du verbe, mais on s'attendrait à ce que la liaison se fasse de la même façon qu'entre le verbe et un vrai COD. Or, les liaisons indiquées ci-dessus nous semblent plus marquées, mais les données disponibles ne permettent pas de confirmer cette intuition quantitativement.

\section{Liaison et élision}

Nous examinerons maintenant les propriétés du bord droit de la combinaison verbe-pronom. En fonction de la forme du pronom inversé et de son contexte à droite, plusieurs phénomènes de sandhi externe («phonétique syntaxique ») sont susceptibles d'être observés.

\subsection{Liaison}

La liaison entre le pronom inversé et le mot suivant semble possible pour les formes -nous et -vous :

- Sommes-nous $[z]$ arrivés ?

- Avez-vous $[z]$ entendu ce bruit ?

Cette liaison facultative est classée «peu fréquente » par Delattre (1956). Dans ces cas, la consonne de liaison associée au pronom et le [z] désinentiel du verbe sont identiques ( Nous sommes [z] arrivés. Vous avez [z] entendu un bruit. »). Pour les formes -ils et -elles, il y a désaccord entre la consonne de liaison typiquement associée à la troisième personne dans le domaine verbal [t], et la consonne de liaison du pluriel [z] fournie par le pronom. La liaison est beaucoup plus difficile dans ces cas (« très rare » pour Delattre 1956) :

- Existent-elles $[z]$ en réalité ?

- Sont-ils $[\mathrm{z}]$ à jour ?

Au singulier, la liaison après -on est totalement exclue, pour la même raison de désaccord consonantique, et à cause de la voyelle nasale ${ }^{3}$ :

- *Qu'a-t-on [n] appris ?

Un examen préliminaire de corpus oraux annotés du français contemporain ${ }^{4}$ a révélé peu d'exemples de la configuration phono-syntaxique en question, et aucun ne présentait une liaison réalisée. Les études doivent être approfondies, mais il est clair que les tendances ne sont pas les mêmes suivant la position du pronom :

- «Vous avez [z] appris » vs « Avez-vous | appris »

Ces observations ont une conséquence pour l'analyse de la séquence verbe-pronom. Les pronoms clitiques peuvent être traités comme des affixes attachés au verbe fléchi (Miller \& Sag 1997), mais la forme verbale qui résulte de la suffixation d'un pronom enclitique (avez-vous) ne doit pas être traitée de la même façon par les règles de la liaison que le verbe simple (avez).

\section{2 Élision}

L'élision du «schwa » est régulière. Elle concerne, pour les variétés pertinentes, les formes -je et -ce. À la différence des mêmes formes employées en position préverbale, l'élision n'est pas obligatoire (même si elle extrêmement fréquente) et elle n'est pas notée orthographiquement :

- «Puis-je avoir » ([Z(ə)av-]) vs. «*Je avais » 
- « J'avais » vs « *Puis-j'avoir » (mal formé orthographiquement)

La forme -elle présente des propriétés similaires, mais le contraste avec ses emplois préverbaux est moins prononcé. En effet, quand le pronom préverbal elle n'est pas clitique, il garde son schwa.

Ces exemples confirment que si la liaison et l'élision sont déclenchées par les mêmes types de contextes à droite - les mots à initiale vocalique, avec des exceptions lexicales (les mots en $h$ aspiré) - l'élision est une processus plus «tardif», c.-à-d. plus purement phonologique et moins sujet aux conditions syntaxiques que la liaison.

La réduction de $t u$ que l'on trouve en position préverbale dans les registres informels n'est pas possible pour - $t u$ inversé :

- «T'avais peur » vs « Vas-tu avoir peur ? *Vas-t'avoir peur? »

Cette observation n'a rien d'étonnant, l'élision de $u$ n'étant pas un phénomène régulier.

\subsection{Enchaînement}

Les consonnes de liaison (dans le cas de -vous, -nous) sont normalement enchaînées, c.-à-d. prononcées comme l'attaque de la première syllabe du mot suivant à initiale vocalique. La possibilité (et le cas échéant le conditionnement) du non-enchaînement de la consonne n'ont pas été étudiés en détails. La base de données orales PFC ne contient aucun exemple de ce type de liaison (avec ou sans enchainement) et les exemples construits semblent très mauvais :

- *Avez-vous [z] | appris ?

Les consonnes fixes des pronoms syllabiques (le [1] de -il(s), -ell(e(s))) sont normalement, mais pas obligatoirement, enchaînées. Il ne s'agit pas d'une particularité des structures inversées. Le nonenchaînement des pronoms non syllabiques -je et -ce nous semble encore moins marqué :

- Est-ce | utile ?

\section{L'inversion simple et complexe}

Dans l'inversion pronominale simple, le pronom postposé est la seule réalisation du sujet. Sans se prononcer sur le statut morpho-syntaxique du pronom (élément syntaxique ou suffixe lié), on peut dire que le pronom réduit la valence syntaxique du verbe. Si un verbe fléchi comme dort a besoin d'un sujet, ce n'est pas le cas de la combinaison dort-elle.

Ce raisonnement est valable sur le plan descriptif. Certaines analyses abstraites mettraient en jeu une structure syntaxique plus étendue avec des positions qui resteraient vides (ou qui seraient éventuellement remplies par des éléments vides). Dans ce type de cadre théorique, identifier les «besoins » du verbe et savoir si ces besoins sont satisfaits ne relève plus seulement de l'observation directe.

Dans l'inversion pronominale complexe - toujours sur le plan descriptif-, le sujet est exprimé deux fois, par un groupe nominal et par un pronom postposé : «Nathalie dort-elle?». L'analyse de cette construction doit alors répondre à deux questions.

- Si le verbe n'a qu'un seul sujet, lequel des deux expressions nominales, Nathalie ou elle, remplit cette fonction?

- Quel est le rapport entre cette structure et l'inversion simple « Dort-elle ? »?

Étant donné la ressemblance superficielle entre les deux structures, il peut être désirable de pouvoir dériver l'une de l'autre. L'inversion simple serait alors une réalisation de l'inversion complexe (avec la suppression du sujet nominal), ou bien l'inversion complexe serait l'expansion d'une inversion simple. Les analyses existantes soulignent surtout les problèmes posés par ces hypothèses. 
Tout d'abord, la distribution des deux types d'inversion n'est pas tout à fait la même : on trouve l'inversion simple, mais pas l'inversion complexe, dans les incises. Pour Kayne (1972, note 27) les propriétés spécifiques des incises peuvent motiver un traitement totalement indépendant, sans lien avec les autres structures inversées. Mais si la fonction interprétative des incises est bien particulière, les inversions qu'elles contiennent (l'inversion pronominale simple et l'inversion stylistique) ne le sont pas. Pour exclure les exemples agrammaticaux, une contrainte sur l'ordre des mots suffirait : Dans une incise, le verbe fléchi doit apparaître en premier.

- « Je vous déteste » dit Hughes / dit-il / *Hughes dit / *Hughes dit-il.

Cette différence de distribution ne met pas en cause l'idée d'un lien dérivationnel entre l'inversion simple et l'inversion complexe.

\subsection{Interprétation du pronom}

Une différence plus problématique concerne la référence du pronom postposé dans les deux types d'inversion. Dans un inversion simple, le pronom peut être explétif («Pleut-il ? »). A priori, cet exemple n'est pas une inversion complexe réduite, parce que la position nominale dans cette structure ne peut être occupée que par un GN (y compris les pronoms forts). Aucune source imaginable n'est bien formée :

- *Il pleut-il ? *Lui pleut-il ? *Le ciel pleut-il ?

Dans l'autre sens, il y a des inversions complexes qui ne peuvent pas être traitées comme l'expansion d'une inversion simple. Il s'agit, entre autres, d'exemples avec un sujet indéfini :

- Tout est-il prêt ? Quelqu'un va-t-il m'aider? Une collaboration est-elle envisageable ?

Les inversions simples correspondantes («Est-il prêt? Va-t-il m’aider? Est-elle envisageable ? )) n'admettent que l'interprétation anaphorique du pronom. Pour les deux derniers exemples, on pourrait essayer de dériver les structures complexes en situant le sujet nominal dans un segment discursif indépendant, comme s'il s'agissait de deux propositions. À ce moment-là la reprise de l'indéfini par $i l$ ou elle pourrait être justifiée. Toutefois, aucune motivation indépendante (d'ordre prosodique ou syntaxique) ne vient appuyer cette approche. Et la première phrase resterait problématique : tout ne peut jamais être repris par $i l$. Il en va de même pour rien, cela, les sujets infinitifs/phrastiques, et les noms prédicatifs dans les locutions verbales (par ex. prêter assistance, Kayne 1972, p. 82-83). Dans les inversions complexes ayant un sujet nominal de ce type, le pronom inversé n'est pas un élément référentiel, mais plutôt un marqueur d'accord.

Les pronoms négatifs introduisent une autre complication :

- Rien ne va-t-il changer? Personne n'a-t-il répondu ? Aucune solution n'est-elle proposée ?

Mis à part le problème de la référence du pronom inversé, les inversions simples contenues dans ces exemples ( *Ne va-t-il changer? *N'a-t-il répondu ? N'est-elle proposée ? ») sont agrammaticales. Bien entendu, on pourrait envisager une analyse où le marqueur négatif serait inséré au cours de la dérivation à partir d'une source bien formée («Va-t-il changer?, etc. »). Ces exemples montrent en tout cas que le sujet nominal dans l'inversion complexe est pleinement intégré syntaxiquement, puisqu'il participe à la concordance négative.

\subsection{L'inversion et la dislocation à gauche}

Les remarques de la section précédente ont mis en question l'idée de relier l'inversion simple et complexe par une dérivation simple. Elles nous ont également renseignés sur la nature du GN dans l'inversion complexe. Il ne peut pas s'agir simplement d'un élément disloqué, comme dans les exemples suivants, sans inversion :

- Nathalie, elle dort. Nathalie et moi, nous devrions partir / on devrait partir. 
En faisant une inversion simple dans la proposition principale de ces deux exemples, on arriverait à des phrases ressemblant superficiellement à des inversions complexes bien formées :

- Nathalie, dort-elle ? Nathalie et moi, devrions-nous partir / devrait-on partir?

Les arguments présentés contre cette analyse sont d'ordre prosodique et syntaxique. Premièrement, on affirme que l'élément disloqué est marqué par l'intonation et par une rupture prosodique. C'est en effet typique, mais pas systématique. Ces effets peuvent être plus ou moins prononcés, et il arrive qu'un élément syntaxiquement disloqué soit prosodiquement intégré dans la phrase (Barnes 1985). Deuxièmement, l'élément disloqué n'occuperait pas la même position syntaxique que le sujet nominal dans une inversion complexe. Les interrogatives totales ne permettent pas d'illustrer la distinction. Il faut un syntagme interrogatif antéposé pour rendre visible l'articulation de la périphérie gauche de la phrase. Jones (1996) donne les exemples suivants :

- Pourquoi cela te gêne-t-il ? ${ }^{*}$ Cela pourquoi te gêne-t-il ?

- Cela pourquoi ça te gêne ? *Pourquoi cela ça te gêne ? [jugements de Jones]

Les deux premières phrases sont des inversions complexes; le sujet nominal cela apparaît obligatoirement après le syntagme qu-. Dans les deux dernières phrases, cela est disloqué à gauche. Il est vrai que la position la plus naturelle pour cela est à la périphérie gauche de la phrase, mais en vérité la dernière phrase ne peut pas être jugée catégoriquement agrammaticale.

Kayne (1972, p. 84) adopte la même contrainte que Jones : Un élément disloqué ne peut pas apparaître entre le syntagme interrogatif et le verbe. La phrase suivante doit donc être dérivée autrement (avec des conséquences importantes pour son analyse de l'inversion complexe) :

- Pourquoi Jean et moi ne devrait-on pas partir tout de suite ?

La même phrase est citée par Kayne (1983, note 9), mais cette fois-ci c'est bel et bien un exemple de dislocation à gauche (sans rupture prosodique) en combinaison avec l'inversion simple. Il nous semble effectivement impossible d'invalider cette dérivation, et la même analyse serait en principe disponible pour une grande partie des inversions complexes.

Cependant, la dislocation à gauche n'est pas à la source de toutes les inversions complexes. Les pronoms tout et rien, par exemple, ne peuvent pas être disloqués de cette façon :

- *Tout, il est prêt. *Rien, il ne va (pas) changer.

En fait, toutes les expressions citées dans la section précédente qui ne permettent pas la reprise par un pronom anaphorique sont naturellement exclues. Certaines d'entre elles peuvent être reprises par ce dans les structures disloquées, ce qui n'explique pas la forme $i l$ qui apparaît dans l'inversion complexe :

- Cela, c'est bien vrai / *il est bien vrai. Cela est-il bien vrai ?

- Abandonner son poste, c'est / *il est envisageable. Abandonner son poste est-il envisageable ?

Il faut admettre, donc, une autre source pour l'inversion complexe, indépendante de la dislocation à gauche. Cela implique que, sans contraintes supplémentaires, beaucoup de phrases auront deux analyses syntaxiques distinctes. Dans la mesure où ces analyses sont associées à des interprétations et à des réalisations prosodiques distinctes, cette ambiguité n'est pas gênante. On peut avoir des réticences à cet égard concernant, par exemple, la phrase de Kayne, mais en l'absence de jugements définitifs, il nous semble prudent de maintenir l'hypothèse de l'ambiguïté et de proposer une seconde analyse de ce type de phrase en tant qu'inversion complexe.

\subsection{Les propriétés du sujet nominal}

Le sujet nominal dans l'inversion complexe présente une partie des propriétés d'un sujet ordinaire. Comme nous l'avons déjà vu, il est intégré pleinement dans la structure de la phrase. Il participe à la concordance négative : personne et rien requièrent la présence du marqueur verbal ne et ils sont 
incompatibles avec pas. Il peut être défini ou indéfini, et ce dernier cas montre que le pronom postposé il ou elle est réduit à une marque d'accord.

Les groupes verbaux (infinitifs et phrases entières) sont acceptables dans cette position, mais les groupes prépositionnels ne le sont pas. Les mêmes restrictions s'appliquent aux sujets ordinaires :

- Sous quelles conditions abandonner son poste est-il envisageable? cf. Abandonner son poste est envisageable sous certaines conditions.

- Que Jacques ait dit cela ne vous intéresse-t-il pas ? cf. Que Jacques ait dit cela vous intéresse.

- *Pourquoi dans le placard est-il une bonne cachette ? cf. *Dans le placard est une bonne cachette pour plusieurs raisons.

Ordinairement, les pronoms personnels forts ne sont pas possibles en position de sujet ("*Moi devrais souffrir »). L'exemple suivant est donc à traiter comme une inversion simple avec un élément disloqué (voir section précédente) :

- Pourquoi, moi, devrais-je souffrir ? ( $\leftarrow$ Moi, je devrais souffrir.)

La liaison entre le sujet nominal et le verbe dans les inversions complexe semble respecter les mêmes conditions que dans une phrase sans inversion. En général, cette liaison est très rare, sauf avec tout :

- Tout [t] est prêt. Tout [t] est-il prêt ?

Delattre (1955) accepte la liaison en [z] après un sujet nominal pluriel :

- Les enfants $[\mathrm{z}]$ attendront longtemps. Les enfants $[\mathrm{z}]$ attendront-ils longtemps ?

Il attribue le fait que la liaison soit (d'après lui) plus fréquente dans la phrase déclarative que dans l'interrogative à un facteur prosodique (l'intonation de la phrase).

En conclusion, il est préférable d'accorder au sujet nominal dans l'inversion complexe le même statut qu'un sujet ordinaire. Mais ce choix rendrait difficile une analyse unitaire des deux types d'inversion, puisque dans l'inversion simple, le sujet ne peut être que le pronom postposé. On n'expliquerait pas non plus le fait que, dans l'inversion complexe aussi, le pronom postposé a des propriétés de sujet. Les exemples de Kayne confirment que quand les traits d'accord du sujet nominal et du pronom divergent, c'est le pronom qui détermine l'accord du verbe et de pronom réfléchi :

- Pourquoi Jean et moi ne s'amuserait-on pas à la soirée ${ }^{6}$

- *Jean et moi s'amuserait. *Pourquoi Jean et moi ne nous amuserions-on pas à la soirée ?

Nous revenons donc au «problème des deux sujets » qui préoccupe tous les auteurs travaillant sur cette question. D'une manière ou d'une autre, il faut dire qu'il y a deux sujets dans l'inversion complexe. La solution astucieuse de Kayne (1972) est de dédoubler tous les nominaux en français : en gros, à chaque fois qu'on introduit un GN, on introduit en même temps le clitique sujet correspondant, dans une structure [GN SCL]. Dans l'écrasante majorité des cas, il faut supprimer l'un ou l'autre élément de la paire avant d'arriver à la structure de surface, mais dans l'inversion complexe, le GN est réalisé normalement, et le clitique subit la même règle de déplacement que dans l'inversion pronominal simple, et se trouve postposé au verbe.

Cette analyse est évidemment incompatible avec les principes de la grammaire transformationnelle (établis plus tard) concernant l'assignation des cas et des $\theta$-rôles. Dans l'analyse de Kayne (1983), les " deux sujets » ne sont plus introduits en même temps. Le sujet nominal est le vrai sujet «profond ", qui se déplace vers la gauche (tout comme le verbe), tandis que le pronom est un explétif inséré dans la position libérée par le sujet nominal, et ensuite cliticisé au verbe ${ }^{7}$ :

- [s cela est faux]

$\rightarrow$ est $_{\mathrm{i}}$ [s cela $t_{\mathrm{i}}$ faux] (verbe à gauche)

$\rightarrow$ cela $_{\mathrm{j}}$ est $t_{\mathrm{i}}\left[\mathrm{s} t_{\mathrm{j}} t_{\mathrm{i}}\right.$ faux] (sujet à gauche) 
Selon Rizzi \& Roberts (1990), cette analyse est toujours incompatible avec les principes de cyclicité et les contraintes sur l'insertion d'éléments au cours de la dérivation. Pour « mettre à jour » l'analyse de Kayne, ils adoptent un modèle plus récent dans lequel il y a deux positions pour le sujet dans toutes les phrases, l'une à l'intérieur de VP et l'autre au niveau supérieur, IP. Dans une phrase simple, le sujet est généré dans VP dans la structure profonde et se déplace à gauche vers l'autre position (afin de recevoir le cas nominatif). Dans l'inversion complexe, sujet nominal est dans VP, comme d'habitude, mais la position supérieure est déjà occupée, dès la structure profonde, par un pronom explétif. Comme dans l'analyse de Kayne (1983), le verbe se déplace à gauche, le sujet nominal se déplace encore plus à gauche, et le pronom, qui se trouve maintenant directement à la droite du verbe, se cliticise à celui-ci.

- [IP $\mathrm{il}_{\mathrm{i}}\left[\mathrm{VP}\right.$ Jean $\mathrm{i}_{\mathrm{i}}$ a mangé]]

$\rightarrow\left[\mathrm{CP}_{\mathrm{j}} \mathrm{a}_{\mathrm{j}}\left[\mathrm{iP} \mathrm{il}_{\mathrm{i}} t_{\mathrm{j}}\right.\right.$ [vP Jean $t_{\mathrm{j}}$ mangé]]] (verbe à gauche)

$\rightarrow\left[\mathrm{CP} \mathrm{Jean}_{\mathrm{i}} \mathrm{a}_{\mathrm{j}}\left[\mathrm{IP} \mathrm{il}_{\mathrm{i}} t_{\mathrm{j}}\left[\mathrm{vP} t_{\mathrm{i}} t_{\mathrm{j}}\right.\right.\right.$ mangé]]] (sujet à gauche)

$\rightarrow\left[\right.$ [CP Jean ${ }_{\mathrm{i}} \mathrm{a}_{\mathrm{j}} \mathrm{t}-\mathrm{t}-\mathrm{i} \mathrm{i}_{\mathrm{i}}\left[\mathrm{IP} t_{\mathrm{i}} t_{\mathrm{j}}\left[\mathrm{vP} t_{\mathrm{i}} t_{\mathrm{j}}\right.\right.$ mangé]]] (cliticisation)

Rizzi \& Roberts expliquent les avantages de leur formulation, mais sans entrer dans les détails un peu techniques du cadre formel adopté, on peut dire que cette analyse et celle de Kayne (1983) sont plus ou moins équivalentes empiriquement. En outre, la structure profonde que posent Rizzi \& Roberts n'est guère motivée intuitivement.

Dans les deux dérivations que nous venons de voir, le pronom $i l$ doit être explétif, afin d'éviter une violation du $\theta$-critère et de construire avec le sujet nominal une «chaîne » bien formée. Les motivations descriptives, en revanche, sont plutôt minces. Si le $i l$ explétif est une notion bien établie, les emplois explétifs de ils/elle(s) sont beaucoup moins évidents. Inversement, on est obligé de dire que le pronom ce n'est pas explétif, malgré ses emplois impersonnels, puisqu'il ne participe pas à l'inversion complexe.

Le problème de l'accord du verbe est laissé de côté dans les travaux récents. Il est vrai que les exemples donnés ci-dessus (avec le sujet coordonné Jean et moi repris par on inversé) représentent l'unique configuration où la question se pose. Pour Kayne (1972) il s'agit donc d'un argument crucial et déterminant; pour Kayne (1983) c'est une anomalie vite évacuée dans une note (dans laquelle précisons-le - il esquisse tout de même deux solutions). On reconnaîtra volontiers la difficulté du problème (voir la section 3.4.4).

Finalement, les analyses de Kayne (1983) et de Rizzi \& Roberts abandonnent l'objectif de faire un lien entre l'inversion complexe et l'inversion simple. Dans l'inversion simple, le pronom est normalement référentiel (argumental). Il serait donc présent dans la structure profonde pour Kayne (au lieu d'être inséré par une règle), et il occuperait probablement la position du sujet profond (dans VP) pour Rizzi \& Roberts.

\subsection{L'analyse compositionnelle}

Dans cette dernière section, nous présentons notre analyse syntaxique de l'inversion complexe. Le cadre formel adopté est une version très simplifiée de HPSG (La grammaire syntagmatique guidée par les têtes, Pollard \& Sag 1994).

\subsubsection{L'inversion simple}

Nous commençons par préciser l'analyse syntaxique de l'inversion simple. Le pronom représente un véritable sujet : il réduit la valence syntaxique du verbe à condition de correspondre aux attentes du verbe. Par exemple, le pronom nous ne satisfait pas les contraintes de dort, et elle ne satisfait pas celles de pleut. Mais dort-elle est une combinaison légitime et recevra l'analyse suivante : 


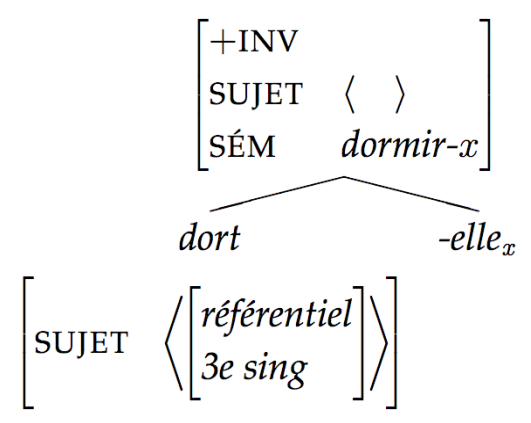

Dans cette représentation, le sujet attendu par le verbe est identifié par le trait SUJET. Si le verbe a besoin d'un sujet syntaxique, il spécifie les propriétés grammaticales de celui-ci (notamment ses traits d'accord et sa référentialité). Dans le cas de dort, le sujet doit être référentiel (par opposition à explétif) et à la $3^{\mathrm{e}}$ pers. sing. Le pronom elle remplit ces conditions. Il peut donc se combiner avec le verbe, et dans la représentation du nœud supérieur, qui encode les propriétés du syntagme ainsi construit, il n'y a plus d'élément dans SUJET. Le syntagme dort-elle n'attend plus de sujet. Le trait SEM encode de façon extrêmement schématique l'interprétation sémantique de la combinaison : elle est bien interprété comme le sujet référentiel du verbe. Le trait + INV sert à signaler la présence d'une inversion pronominale pour la syntaxe externe de la combinaison. Par exemple, la conjonction si se combine avec une phrase complète, à condition qu'elle porte le trait -INV. Ce serait le cas de elle dort ( $\rightarrow$ « si elle dort ») mais pas de dortelle $(\rightarrow$ "*si dort-elle »). La combinaison dort-elle peut aussi constituer une phrase complète à elle seule. La représentation du nœud supérieur est compatible avec celle d'une phrase indépendante.

Dans cette première analyse, le pronom est référentiel, mais un verbe peut également exiger un sujet explétif. Voici l'analyse de la combinaison pleut-il :

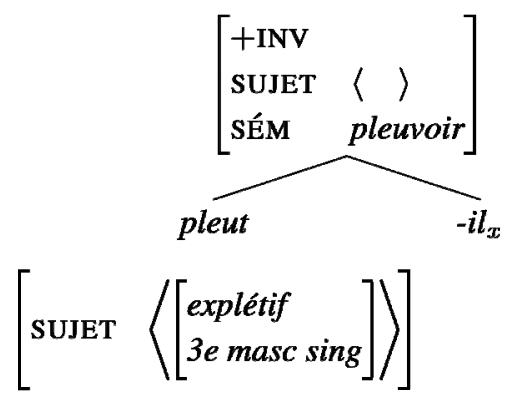

Ici, le pronom il réalise le sujet explétif du verbe impersonnel. Il n'est pas représenté dans la partie sémantique (autrement dit, il ne reçoit pas de $\theta$-rôle du verbe), mais il a tout de même un indice $x$, auquel sont associés ses traits d'accord (personne, nombre, genre). Au nœud supérieur, la valence syntaxique du verbe est réduite : pleut-il n'a plus besoin d'un sujet.

Ces deux exemples relèvent d'un schéma général pour l'inversion simple qu'on peut définir en précisant les contraintes suivantes :

- La branche gauche est un verbe fléchi, portant le trait SUJET $<S>$ ( $S$ est un ensemble de propriétés grammaticales).

- La branche droite est un pronom faible inversé, dont les propriétés sont compatibles avec $S$.

- Le résultat de la combinaison porte les traits + INV et SUJET $<>$ (valeur vide).

L'accord du verbe est déterminé déjà au niveau lexical. Par exemple, comme nous l'avons vu, la forme fléchie dort spécifie qu'elle attend un sujet de la $3^{\mathrm{e}}$ pers. sing. La forme dorment aurait la valeur SUJET $<$ [3e plur $]>$, et la forme dormais la valeur SUJET $<[1$ re/2e sing $]>$. Le schéma de combinaison vérifie, au moment de combiner le verbe avec le pronom, que les propriétés du pronom s'accordent bien avec les 
spécifications du verbe. Cette vérification échoue pour *dort-nous et *dormais-ils, par exemple, et ces combinaisons sont rejetées.

Au niveau sémantique, l'association de l'argument externe du prédicat avec le sujet est également déterminée lexicalement (le cas échéant). Étant donné que cet aspect de l'analyse n'est pas central dans notre discussion, nous nous contenterons d'une approche intuitive de la structure argumentale, sachant que les principes d'association des rôles sémantiques et de composition sémantique sont explicitement formalisés dans ce modèle.

La restriction sur la catégorie grammaticale de l'élément à droite exclut les combinaisons dort Nathalie (Nathalie n'est pas un pronom) et dort lui (lui est un pronom fort). Ce schéma n'est pas destiné à analyser l'inversion nominale, qui obéit à des contraintes bien distinctes.

La précision "pronom inversé » est nécessaire pour rendre compte des faits morpho-phonologiques décrits dans la section 1.2. Dans parle-t-elle, par exemple, ce n'est pas la forme préverbale qui est sélectionnée $\left({ }^{*}[\mathrm{parlEl}]\right)$, mais obligatoirement l'allomorphe $\left[\mathrm{tEl}{ }^{8}{ }^{8}\right.$. Nous laisserons de côté la formalisation des autres effets morpho-phonologiques associés à l'inversion pronominale.

\subsubsection{Variante de l'inversion simple}

Nous introduisons maintenant une ambiguïté systématique dans l'analyse des séquences verbe-pronom de l'inversion simple. Le pronom peut réaliser le sujet du verbe et réduire sa valence, comme dans les analyses de la section précédente, ou bien il peut laisser cette valence inchangée. Considérons cette deuxième analyse pour la combinaison dort-elle :

$$
\begin{aligned}
& \underbrace{\left.\left[\begin{array}{ll}
+\mathrm{INV} & \\
\text { SUJET } & \left\langle\left[\begin{array}{l}
\text { référentiel } \\
\text { 3efém sing }
\end{array}\right]\right\rangle \\
\text { SÉM } & \text { dormir-? }
\end{array}\right]\right\rangle}_{\text {dort }} \\
& {\left[\text { SUJET }\left\langle\left[\begin{array}{l}
\text { référentiel } \\
3 e \text { sing }
\end{array}\right]\right\rangle\right]}
\end{aligned}
$$

Dans cet exemple, on fait toujours la vérification des propriétés grammaticales du pronom (traits d'accord, référentialité), qui doivent être compatibles avec les attentes du verbe. Mais cette fois-ci la réalisation du pronom ne supprime pas la valeur SUJET du verbe. Cette valeur devient plus spécifique : les propriétés du pronom sont ajoutées aux contraintes initialement imposées par le verbe. Le verbe dort ne spécifie pas a priori le genre de son sujet, mais la combinaison avec le pronom elle fait apparaitre la contrainte supplémentaire «fém » dans la valeur SUJET du nœud supérieur. Dans la partie sémantique, l'identité de l'argument externe du prédicat dormir est toujours inconnue. Par conséquent, à la différence de la première analyse dans la section précédente, cette combinaison ne peut pas constituer une phrase complète et indépendante.

La définition de ce schéma doit inclure les contraintes suivantes :

- La branche gauche est un verbe fléchi, portant le trait SUJET $\langle S>$.

- La branche droite est un pronom faible inversé, dont les propriétés $P$ sont compatibles avec $S$.

- Le résultat de la combinaison porte les traits $+\mathrm{INV}$ et $\operatorname{SUJET}\langle S+P>$.

L'élément à gauche doit être un verbe simple. L'application répétée du schéma est ainsi exclue : *dortelle-elle. 
Dans les grammaires HPSG, la réalisation du sujet (même par un pronom) devrait normalement entrainer la suppression de l'élément correspondant dans SUJET. C'est la conséquence d'une contrainte globale, le Principe de Valence. La définition de ce schéma est donc exceptionnelle, même si elle ne pose aucun problème technique majeur. Une autre possibilité serait de supprimer la valeur SUJET, conformément au Principe de Valence, et d'en reconstruire une nouvelle au noud supérieur. Ce n'est en réalité qu'une interprétation procédurale du même schéma, qui n'enlève en rien la difficulté conceptuelle. En effet, l'introduction de nouveaux éléments de valence au cours de la dérivation constitue tout autant une violation du principe en question. Toutefois, nous serons amenés à définir une version spéciale de ce schéma pour traiter les particularités du pronom on (voir la section 3.4.4), et dans ce cas précis une division en deux étapes - la suppression de SUJET suivie de l'introduction d'une nouvelle valeur SUJET — présenterait des avantages.

Dans les autres cas, on pourrait mettre les deux schémas pour l'inversion simple en rapport avec un type d'analyse qui se rencontre en LFG (La grammaire lexicale fonctionnelle, Dalrymple 2000). Dans les grammaires de ce cadre formel, les pronoms ont souvent un double statut. Ils peuvent contribuer leur valeur «prédicative» (c.-à-d. leur sémantique référentielle) ou bien ils peuvent ne contribuer que des informations formelles (traits d'accord), en attendant qu'une autre expression nominale vienne instancier la valeur prédicative. C'est l'approche mise en œuvre notamment dans l'analyse du redoublement clitique, un phénomène assez semblable à l'inversion complexe. Nos deux schémas correspondraient en fait à une seule règle $\mathrm{LFG}$, et une entrée lexicale pronominale (clitique) précisant l'ambiguité prédicative :

$$
\begin{aligned}
& \text { - } \mathrm{VP} \rightarrow \mathrm{V} \\
& \uparrow=\downarrow \quad(\uparrow \mathrm{SUBJ})=\downarrow \\
& \text { - } \mathrm{Cl} \rightarrow \quad(\uparrow \text { PRED })=\left({ }^{\prime}\right. \text { PRO') }
\end{aligned}
$$

Nous fermons à présent cette parenthèse pour poursuivre l'analyse de l'inversion complexe en HPSG.

\subsubsection{L'inversion complexe}

Le schéma défini dans la section précédente produit des structures incomplètes, destinées à devenir des inversions complexes. La première étape de la dérivation de l'inversion complexe est donc une variante de l'inversion simple, ce qui répond à notre objectif global de relier les deux types d'inversion.

La seconde étape de la dérivation est la réalisation du sujet nominal. Comme il a été démontré dans la section 3.3, le sujet nominal dans une inversion complexe a les même propriétés que le GN sujet d'une phrase ordinaire. Nous n'introduisons donc aucune règle nouvelle; il s'agit du même schéma de combinaison utilisé pour réaliser le sujet dans une phrase ordinaire (par ex. « Nathalie dort »).

- La branche droite est une projection verbale portant les traits SUJET $<S>$ et $\alpha$ INV.

- La branche gauche est un $\mathrm{GN}^{9}$, dont les propriétés sont compatibles avec $S$.

- Le résultat de la combinaison porte les traits SUJET < > et $\alpha$ INV.

Voici l'analyse de l'inversion complexe Nathalie dort-elle? : 


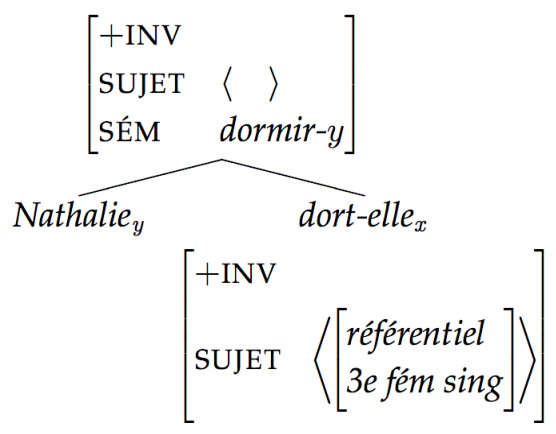

Conformément à la définition du schéma, le GN sujet Nathalie apparaît à gauche. La compatibilité de ses traits grammaticaux avec les spécifications du composé verbal dort-elle est vérifié. Au niveau supérieur, la valence est réduite (le trait SUJET ne contient plus d'élément), et Nathalie est identifiée comme l'argument externe du verbe. Cette structure peut maintenant constituer une phrase complète, ou bien être insérée dans une structure plus étendue, à condition que le trait +INV soit approprié (par ex. « Où Nathalie dort-elle ? » mais «*Si Nathalie dort-elle »).

La combinaison *Les femmes dort-elle est rejetée au même titre que *Les femmes dort: Les traits du GN sujet ne s'accordent pas avec les contraintes imposées lexicalement par le verbe dort. La combinaison *L'homme dort-elle est rejetée (à la différence de la combinaison grammaticale L'homme dort) parce que le pronom inversé elle ajoute la spécification «fém » aux contraintes imposées au sujet.

Ce schéma exclut les pronoms faibles (qui n'ont pas le statut de GN) : *Il dort-il ? *C'est-il vrai ? Les structures grammaticales $I l$ dort et C'est vrai sont à analyser à l'aide d'un autre schéma. La phrase Elle dort-elle? est en principe acceptée, dans la mesure où le pronom elle peut être une forme forte, avec le statut de GN (cf. Lui dort-il ?, également accepté).

Dans le cas de pleut-il, la double analyse du pronom postposé reste possible, mais cette inversion simple ne pourra jamais devenir une inversion complexe. La combinaison pleut-il peut avoir une valeur sujet, avec la spécification "explétif». Mais dans le registre stylistique pertinent, le seul sujet possible est le pronom faible $i l$, qui ne remplit pas les conditions pour entrer dans un schéma GN + verbe : *Il pleutil $?^{10}$.

Nous ne proposons pas une analyse complète de l'inversion stylistique, mais il y a lieu de faire une remarque sur l'interaction des phénomènes. Il s'agit de définir un autre schéma de combinaison pour la réalisation d'un GN sujet, cette fois-ci à la droite du verbe («(Où) dort Nathalie ? »). Dans la formulation de ce schéma, il faudra préciser que le verbe porte le trait -INV. Cette contrainte bloquera la co-occurrence de l'inversion pronominale et stylistique dans une même phrase : «*Où dort-elle Nathalie? »

\subsubsection{Extensions}

L'analyse proposée n'est pas encore en mesure de traiter les cas problématiques signalés dans les sections 3.1-3.3.

- Certaines expressions (cela, GV infinitifs, phrases) admettent la reprise anaphorique par ce ; or ce est exclu dans l'inversion complexe.

- Certaines expressions (celles du point précédent, plus tout, rien, noms prédicatifs, ...) n'admettent pas la reprise anaphorique par il/elle; or elles apparaissent avec il/elle dans l'inversion complexe.

- Un sujet de la $1^{\text {re }}$ pers. plur. peut apparaître avec on dans l'inversion complexe.

Il est donc clair que les deux sujets dans une inversion complexe ne sont pas reliés par la même relation anaphorique qui existe, par exemple, entre un élément disloqué à gauche et sa reprise pronominale dans la phrase. On peut contraster la « coréférence » dans un cas (dislocation + reprise), avec la « coindexation » dans l'autre (inversion complexe). Des principes distincts régissent le choix du pronom dans les deux cas, 
même si le plus souvent (en dehors des points en question) ils arrivent au même résultat. Les termes sont choisis pour opposer d'un côté l'identification de deux référents sémantiques (coréférence) et de l'autre côté l'identification des traits formels de deux expressions syntaxiques (coindexation).

Dans cette optique, un pronom peut référer à une classe d'entités sémantiques, sans forcément partager les traits formels de toutes les expressions syntaxiques correspondant à ces entités. Ou bien il peut partager les traits formels d'autres expressions correspondant à des entités auxquelles il n'est pas capable de référer. Formellement, nous proposons de suivre l'approche de Wechsler \& Zlatić (2003), en associant à chaque expression deux indices distincts : «REFERENT » et « INDEX ». Dans l'inversion complexe, les deux sujets ont des valeurs INDEX identiques (ou plus précisément, unifiables). La valeur INDEX correspond tout simplement à l'ensemble des traits formels personne, nombre et genre. Dans la définition du dernier schéma de combinaison dans la section précédente, la vérification de la compatibilité des traits d'accord du GN sujet est donc une opération de coindexation. Accessoirement, le GN sujet et le pronom inversé peuvent avoir le même REFERENT, mais ce n'est pas une condition imposée par la construction.

La valeur INDEX de la plupart des pronoms est évidente (il $=[3$ e masc sing $]$, elles $=[3$ e fém $\mathrm{plur}], t u=[2 \mathrm{e}$ sing], etc.), tout comme celle des expressions nominales (Nathalie $=$ [3e fém sing], tout $=$ [3e masc sing], assistance $=[3$ e fém sing $]$, etc.) La coindexation est donc vérifiée dans les exemples suivants :

- Nathalie dort-elle ?, Tout est-il prêt ?, Assistance est-elle prêtée ?

À noter toutefois que le sujet nominal et le pronom inversé ne sont pas coréférentiels dans les deux derniers exemples (c.-à-d. valeurs REFERENT distinctes). Les cas de coindexation échouée sont correctement exclus :

- *Nathalie dorment-elles ?, *Tout es-tu prêt ?, *Assistance est-il prêtée ?

Le pronom ce est plus problématique. Il a une grande capacité référentielle, mais à en juger par son absence totale dans l'inversion complexe, il ne peut jamais être coindexé avec une autre expression. Dans notre système, cela se traduit par une valeur REFERENT très générale et une valeur INDEX maximalement spécifique, autrement dit unique. En même temps, les combinaisons grammaticales $c$ 'est et est-ce fixent les valeurs de personne ( $3 e$ ) et de nombre (sing), car ces spécifications apparaissent dans la valeur SUJET du verbe fléchi est. Il ne reste plus que le trait genre, pour lequel nous proposons la valeur unique neut. C'est un choix plutôt traditionnel, mais il faut reconnaitre son caractère ad hoc, d'autant plus que les besoins de l'analyse nous obligent à poser la valeur masc pour toutes les expressions apparentées : cela, $c ̧ a$, les GV infinitifs, les arguments phrastiques. Une fois mises en places, ces valeurs conduisent aux résultats voulus :

- *Cela est-ce vrai ? *Abandonner son poste est-ce envisageable ? (INDEX [3e neut sing]) ${ }^{11}$

- Cela est-il vrai ? Abandonner son poste est-il envisageable ? (INDEX [3e masc sing])

Enfin, la relation de coindexation est réflexive, mais *C'est-ce vrai ? n'est pas généré, car la forme faible ce n'a pas le statut de GN.

À notre sens, la solution de Kayne (1983) au problème de $c e$ n'est pas moins artificielle. Dire qu'il est obligatoirement référentiel l'exclut catégoriquement de l'inversion complexe, mais il faut alors démontrer sa référentialité dans tous ses autres emplois.

Finalement, nous répondons au dernier point problématique : le pronom on. Rappelons la phrase de Kayne :

- Pourquoi Jean et moi ne devrait-on pas partir tout de suite ?

Cet exemple ne peut pas être analysé comme une inversion complexe si on exige la simple coindexation du GN sujet Jean et moi (INDEX [1re masc plur]) et du pronom on (INDEX [3e sing]). En plus, étant donné cette valeur INDEX (déterminée par l'accord dans devrait-on), on s'attendrait à une concurrence entre $i l$ et on inversés, mais il n'en est rien :

- *Tout est-on prêt? *Quelqu'un dort-on? 
Dans les inversions complexes, on apparaît exclusivement avec des GN à la $1^{\text {re }}$ pers. plur., alors que dans l'inversion simple, il garde toutes ses interprétations (sujet indéfini, $2^{\mathrm{e}}$ pers., $1^{\mathrm{re}}$ pers. plur., etc.) Nous proposons donc que pour cette forme, le schéma de l'inversion simple défini dans la section 3.4.2 n'est pas une simple variante du schéma de 3.4.1. Dans ce cas très particulier, la valeur SUJET de la combinaison verbe + pronom n'est pas déterminée en fonction des propriétés des branches $(<S+P\rangle$ dans la définition du schéma). La compatibilité de l'INDEX du pronom on ([3e sing]) avec la valeur SUJET du verbe est vérifiée (afin de valider l'accord du verbe) mais au nœud supérieur la valeur SUJET n'est pas calculée, mais construite : [1re plur]. Nous avons déjà évoqué dans la section 3.4.2 une analyse en deux étapes pour on inversé : réalisation/suppression de la valeur SUJET initiale, ensuite introduction de la nouvelle valeur SUJET. La combinaison devrait-on est alors prête à recevoir le GN sujet Jean et moi à la prochaine étape de la dérivation.

Les exemples détaillés présentés dans cette partie analytique contiennent tous un verbe intransitif, mais l'analyse peut s'adapter à des structures de complémentation plus complexes. En particulier, d'autres types d'accord pronominal sont envisageables. Morin (1985) donne les exemples suivants, où le pronom inversé s'accorde avec un pronom clitique à l'accusatif :

- \%Cela la gêne-t-elle ? \%Comment Pierre la trouve-t-elle ?

Bien entendu, l'importance qu'il faut attacher aux exemples de ce type est discutable, mais le système d'accord qu'ils représentent est facilement analysable dans l'approche proposée : il suffit de formuler une variante de la deuxième règle pronominale dans laquelle les traits du pronom inversé s'ajoutent non pas aux contraintes sur le sujet, mais aux contraintes sur le complément (encodées dans un autre trait de valence COMPLEMENTS).

\section{Conclusion}

L'inversion pronominale est un phénomène fondamentalement syntaxique qui soulève aussi des questions concernant l'interface de la syntaxe avec la morphologie et la phonologie. Dans cet exposé nous avons examiné plusieurs aspects de l'inversion. La perspective diachronique permet de mieux comprendre le processus de grammaticalisation (la fusion morpho-syntaxique d'une séquence verbe-pronom) qui a produit les formes que l'on observe aujourd'hui. Ensuite nous avons décrit les propriétés phonosyntaxiques des constructions inversées (surtout la possibilité de faire la liaison après le pronom inversé). Cet aspect du phénomène est peu étudié, et nous n'avons pu présenter ici que quelques observations préliminaires.

Enfin nous nous sommes concentrés sur l'analyse formelle de l'inversion complexe. Nous avons réexaminé la relation entre l'inversion complexe et la dislocation à gauche. L'absence de jugements catégoriques nous a amenés à maintenir une ambiguïté structurale pour une grande partie des exemples. Notre résumé critique des travaux précédents a révélé l'abandon peu motivé de l'objectif initial de relier l'inversion complexe à l'inversion simple. L'analyse formelle proposée rétablit ce lien, mais les cas récalcitrants classiques (à savoir les pronoms inversés $c e$ et on) ne permettent toujours pas un traitement réellement unitaire.

\section{Références bibliographiques}

Barnes, B. K. (1985). The Pragmatics of Left Detachment in Spoken French. Amsterdam : John Benjamins.

Beaulieux, C. (1967). Histoire de l'orthographe française. 2 vol. Paris : H. Champion.

Dalrymple, M. (2001). Lexical Functional Grammar. Syntax and Semantics vol. 34. New York : Academic Press.

Delattre, P. (1956). La Fréquence des liaisons facultatives en français. The French Review 30:1, pp. $48-54$.

Delattre, P. (1955). Les facteurs de la liaison facultative en français. The French Review 29:1, pp. 42-49.

Fouché, P. (1967). Morphologie historique du français. Le verbe. Paris : Klincksieck. 
Fouché, P. (1961). Phonétique historique du français. Volume III : Les consonnes et index général. Paris: Klincksieck.

Fouché, P. (1959). Traité de prononciation française. Paris : Klincksieck.

Holbrook, R. T. (1923). Parle on et parle-t-on : pour fixer une date. The Modern Language Journal 8:2, pp. 89-91.

Jones, M. A. (1996). Foundations of French syntax. Cambridge : Cambridge University Press.

Kayne, R.S. (1983). Chains, categories external to S, and French complex inversion. Natural Language \& Linguistic Theory 1:1, pp. 107-139.

Kayne, R.S. (1973). Subject Inversion in French Interrogatives. In J. Casagrande and B. Saciuk (eds), Generative Studies in Romance Languages. Rowley, MA : Newbury House, pp. 70-126.

Labelle, M., \& P. Hirschbühler. (2005). Changes in clausal organization and the position of clitics in Old French. In Batllori, M., et al. (éds.) Grammaticalization and Parametric Change. Oxford : Oxford University Press.

Miller, P. H., \& I. A. Sag. (1997). French clitic movement without clitics or movement. Natural Language and Linguistic Theory 15:3, pp. 573-639.

Morin, Y.-C. (1985). On the two French subjectless verbs voici and voilà. Language 61:4, pp. 777-820.

Pollard, C. J., \& I. A. Sag. (1994). Head-Driven Phrase Structure Grammar. Chicago : University of Chicago Press.

Rizzi, L. and I. Roberts (1990). Complex Inversion in French. Probus 1.1, pp. 1-30.

Wechsler, S. \& L. Zlatić. (2003). The Many Faces of Agreement. Stanford : CSLI Publications

Zink, G. (1986). Phonétique historique du français. $6^{\mathrm{e}}$ édition. Paris : PUF.

Zink, G. (1997). Morphosyntaxe du pronom personnel (non réfléchi) en moyen français (XIV ${ }^{e}-X V^{e}$ siècles). Genève : Droz.

\footnotetext{
${ }^{1}$ Avec l'exception du pronom ce, qui a une distribution très restreinte (surtout en position inversée).

${ }^{2}$ Cette observation n'exclut pas la possibilité de considérer [ $\mathrm{t}$ ] comme une marque de la $3^{\mathrm{e}}$ personne associée à tout le domaine verbal (et non pas spécifiquement au verbe). Cette idée peut expliquer les erreurs du type pas [t]-à moi, vat-en guerre, qui n'ont pas le même statut que va-t-il, alla-t-on.

${ }^{3}$ Selon Fouché (1959), la liaison est possible dans un style soutenu entre -on inversé et un pronom adverbial suivant («Peut-on [n] y répondre?»), mais cette prononciation n'est plus acceptée aujourd’hui.

${ }^{4}$ Phonologie du Français Contemporain : http: //www.projet-pfc.net/

${ }^{5}$ Ex. (48) de Kayne (1972, p. 82).

${ }^{6}$ Ex. $\left(\mathrm{o}^{\prime \prime}\right)$ de Kayne (1972, note 32).

${ }^{7}$ Kayne (1972) rejette avec force cette idée de « copie pronominale ».

${ }^{8}$ Le [t] pourrait aussi être inséré par le schéma de combinaison. Rien ne permet de départager les analyses. L'approche pronominale " prédirait» des erreurs comme Est-elle, ou t-il, arrivé(e) ?, par contraste avec Arrive t-ou repart-elle?, qui favoriserait plutôt l'approche constructionnelle.

${ }^{9}$ Il faut comprendre « GN, GV infinitif, Phrase », c.-à-d. toute expression ayant la distribution d'un sujet non clitique.

${ }^{10}$ «Ça pleut-il » est syntaxiquement bien formé, mais présente évidemment un mélange de registres incompatibles.

${ }^{11}$ Rappelons que la dislocation à gauche est une source possible pour ces exemples, qui donnent lieu en fait à des jugements variables (voir la section 3.2).
} 